\title{
Información y participación ciudadana en el contexto del gobierno abierto: las potencialidades de la biblioteca pública
}

\author{
Alejandro Ramos Chávez \\ Universidad Nacional Autónoma de México - UNAM, México
}

REVIEW

\begin{abstract}
Resumen
Diversos gobiernos alrededor del mundo han establecido tendencias encaminadas a obtener gestiones gubernamentales que impliquen una participación ciudadana más amplia. En este sentido, el gobierno abierto representa una de las tendencias más visibles en la comprensión de la necesidad de contar con ciudadanos mejor informados y más participativos en el desarrollo de los asuntos públicos, al tomar en consideración tres elementos principales: i) la necesidad de contar con mayores niveles de transparencia y rendición de cuentas gubernamentales; ii) el establecimiento de formas de gobierno más plurales y horizontales en un sentido más cercano al concepto de gobernanza; iii) la importancia de la utilización de las herramientas digitales para llevar a cabo los procesos antes referidos. El objetivo del documento es el de poner a consideración la importancia de la información para alcanzar una ciudadanía más involucrada en los asuntos públicos en el contexto de los debates del gobierno abierto, así como el papel que podría tener la biblioteca pública como espacio democrático para la obtención de información y encuentro ciudadano. Las conclusiones apuntan a señalar la importancia, así como los principales retos, que tienen las bibliotecas públicas para constituirse como espacios esenciales del gobierno abierto y la construcción de ciudadanía.
\end{abstract}

Palabras clave

Biblioteca pública; Ciudadanía; Gobierno abierto; Información; Participación ciudadana

Information and citizen participation in the context of open government: the possibilities of the public library

\begin{abstract}
A range of governments around the world have established trends oriented towards government actions that result in greater citizen participation. In this sense, open government represents one of the most viable trends to understanding the need to have better informed and more participative citizens in the development of public matters, taking into consideration three principle factors: i) the need for greater government transparency and accountability; ii) establishment of more plural and horizontal forms of government closer to the concept of governance; iii) the importance of using digital tools to carry out these processes. The aim of this article is to consider the importance of information to achieve a citizenship that is more involved in public matters, within the context of open government debates, as well as the role that the public library could have as a democratic space to obtain information and as a public meeting point. The conclusions indicate the importance, as well as the challenges, that public libraries have in establishing themselves as essential spaces of open government and the construction of citizenship.
\end{abstract}

Keywords

Citizen participation; Citizenship; Information; Open government; Public library 


\section{Introducción}

El concepto de ciudadanía ha sido comprendido desde múltiples puntos de vista y enfoques metodológicos que lo han analizado. Lo anterior, a la par de enriquecer su estudio, ha traído a su vez complicaciones o confusiones interpretativas. En este sentido, en el presente documento se pretende abordar el debate de este concepto, contextualizándolo a la luz de los postulados teóricos del gobierno abierto. Este último concepto surge como un enfoque de gobierno, en el que se persiguen objetivos para hacer más eficiente la gestión de las políticas públicas así como la puesta en marcha de programas y proyectos gubernamentales, es decir, hacer más eficiente el desempeño gubernamental.

Tomando en consideración estos elementos, el presente documento está dividido en cuatro apartados generales. En el primero de ellos se aborda el análisis del concepto de ciudadanía, vinculando la importancia de la información en la construcción ciudadana. En este mismo apartado se realiza un recorrido interpretativo del concepto de ciudadanía, que va desde su visualización más jurídica hasta una visión más participativa y activa del mismo. Sin embargo, para que esa participación pueda darse de forma correcta, surge la necesidad de que los ciudadanos posean información útil y relevante, con objeto de que sus posturas, ideas y visones resulten bien argumentadas y fincadas en conocimiento de causa. De igual forma, se toman en consideración elementos que apuntan a entender al ciudadano desde una nueva perspectiva, que se vincula con el ejercicio ciudadano mediado por el uso de las tecnologías y de forma específica el internet.

En el segundo apartado se toma en consideración, de forma específica, la contextualización de la ciudadanía en el modelo del gobierno abierto, tomando en cuenta su surgimiento teórico así como su uso en el argot político, rescatando los pilares conceptuales que le constituyen. En este sentido se toman en cuenta tres ideas generales que perfilan los objetivos generales del gobierno abierto: En primer lugar se encuentran la transparencia y la rendición de cuentas como prácticas fundamentales que los gobiernos deben de seguir, con objeto de que la ciudadanía esté enterada no sólo de las formas en las que son atendidos los asuntos públicos, sino también de los motivos que tuvieron los funcionarios públicos para tomar esas determinaciones y no otras. Con esta información pública se espera que la ciudadanía, a la par de tener elementos para valorar y evaluar el quehacer gubernamental, cuente con información necesaria para que su participación resulte más y mejor fundamentada. El segundo de los elementos analizados es el de la gobernanza, ya que en la idea del gobierno abierto se visualiza una ciudadanía proactiva en la toma de decisiones públicas, abandonando con ello la forma tradicional de toma de decisiones meramente burocrática, por lo que en esta parte del documento se hace una diferenciación entre los modelos de gobernabilidad y el de gobernanza. El tercer pilar conceptual analizado es el de la utilización de las TIC en principalmente dos dimensiones de análisis. La primera de ellas es la del uso de las tecnologías por parte del gobierno, como una ventana de constante comunicación, así como un espacio por el que se pueda dar de mejor forma a la transparencia y a la rendición de cuentas. La segunda como mecanismos a utilizar por la ciudadanía para llevar acabo tanto los procesos de información, así como de involucramiento y participación en los asuntos públicos.

En el tercer apartado del documento se rescata la importancia de un tema que ha estado al margen de las discusiones del gobierno abierto en algunos países, como lo es el caso de México, relacionado con el valor y la trascendencia de la biblioteca pública como espacio esencial para el desarrollo ciudadano, por ser en sí misma un lugar democrático para el acceso a la información, así como un posible punto de encuentro entre los ciudadanos. En este sentido, rescatando aportes de autores relacionados con el tema, se propone la importancia de la revalorización e impulso de las bibliotecas públicas para el desarrollo ciudadano.

Finalmente, en el último apartado del documento, se desarrollan algunas ideas generales a manera de conclusión.

\section{Antecedentes teóricos de la información y la participación ciudadana}

El concepto de ciudadanía ha transitado de una definición meramente jurídica, al entenderla como el derecho de los individuos a tener derechos y obligaciones por habitar en un determinado Estado Nación, a una visión más amplia que implica un involucramiento ciudadano más amplio en la forma de ejercer los procesos gubernamentales y en general con la forma en la que son atendidos los asuntos públicos. Gran parte de este cambio de paradigma surgió a partir de las numerosas críticas que se dieron alrededor de la visión clásica de ciudadanía, pues se le veía estrechamente vinculada con aspectos racionalistas, individualistas, 
homogeneizadores y universalistas, que no permitían el reconocimiento de las diferencias existentes entre los individuos para el ejercicio pleno de su ciudadanía (Bolos, 2008).

Tomando en consideración lo anterior, existen dos grandes concepciones en las que se puede entender la idea de ciudadanía. En primer lugar en una definición de ciudadanía denominada como de baja intensidad (Bonet, 2009; O’Donnell, 1993), delegativa (Akutsu \& Pinho, 2003; O’Donnell, 1994; Peruzzotti, 1997) o pasiva (Farinós, 2005; Kymlicka \& Norman, 1997), en donde es ciudadano aquella persona que además de contar con derechos y responsabilidades, también participa en los procesos electorales para designar puestos de representación popular, es decir, es ciudadano también el que elige gobierno mediante su voto. En este sentido, algunos autores han encontrado en este tipo de ciudadano a individuos "[...] dotados jurídicamente de derechos, pero desprovistos de la capacidad real y del poder efectivo para ejercerlos plenamente; la despolitización de la población, que ha delegado algunas de las funciones y responsabilidades que tendrían que estar en manos directas de la gente [...]" (Bonet, 2009, p. 14), por lo que resultaría rebasado el uso del concepto únicamente circunscribiéndolo en este plano de participación (Correa \& Noé, 1998; Dasí, 2005; Habermas, 1998; Kymlicka \& Norman, 1997; Valente, 2000). Aun así, en este tipo de ciudadanía que principalmente participa en la elección de sus representantes públicos, exige de ciudadanos bien informados tanto de sus problemáticas sociales así como de las plataformas y propuestas de los diversos candidatos a ocupar puestos de representación popular, con objeto de que su participación misma en las elecciones resulte fundamentada y acorde a la búsqueda de respuestas que atiendan las demandas y problemáticas sociales.

La segunda de las concepciones de la ciudadanía se relaciona más con ciudadanos que constantemente se involucran con la política y con los asuntos públicos. Este tipo de ciudadanía ha sido caracterizada como participativa (Borchorst, Bødker, \& Zander, 2009; Parker, 1989; Vromen, 2003), activa (Fleury, 2003; Habermas, 1998; Kymlicka \& Norman, 1997), sustantiva (Bolos, 2008), de alta intensidad (Bonet, 2009) o deliberativa (Ganuza, 2012). Las diferencias principales entre la ciudadanía de baja intensidad y este tipo de ciudadanía, es que en ésta última los ciudadanos además de participar de forma responsable, es decir, de forma informada y fundamentada en los procesos electorales, participan activamente fuera de los procesos coyunturales de elección de funcionarios públicos; por lo que frecuentemente están enterados, informados y opinan constantemente sobre la gestión de los asuntos públicos y el desarrollo de la política en general. Como se puede vislumbrar, en este tipo de ciudadanía, se requieren mejores canales tanto para la obtención de información, así como más y mejores canales para que los ciudadanos deliberen con sus pares (con otros ciudadanos), generando con ello opinión pública que permita a los funcionarios públicos adecuar sus acciones para alcanzar el bien general, o bien sujetarse al escrutinio público.

Estos cambios en el entendimiento del concepto de ciudadanía, se relacionan también con enfoques que surgieron a partir de la segunda parte del siglo pasado, pero con mucha mayor fuerza a finales de ese mismo siglo y principios del siglo XXI, en donde aspectos fundamentales como la pérdida de centralidad en el uso de canales tradicionales de participación política, como lo fueron los partidos políticos y los sindicatos, dieron pie a nuevas formas de organización y participación ciudadana en temas de interés general (Lechner, 2000; Winocur, 2003a, 2003b). De igual forma, el surgimiento de grupos que no se encontraban plenamente identificados con la noción homogeneizadora de ciudadanía, ya que a partir de sus propias diferencias buscaban su reconocimiento y el derecho a ser escuchados y aceptados, tuvieron auge a partir de la década de los años sesenta del siglo pasado, lo que llevó a grupos de jóvenes, ancianos, mujeres, indígenas, homosexuales, así como a grupos que perseguían un objetivo particular como lo son los grupos para el cuidado medioambiental, de comercio justo o de protección a los animales, por citar algunos, a exigir su ciudadanía a partir de esas particularidades específicas en las que se desenvolvían, buscando ante todo un derecho a ser escuchados a partir del reconocimiento de la pluralidad y diversidad de posturas en la idea de la construcción ciudadana.

Por otro lado, el debate de las formas ciudadanas contextualizándolas a modalidades televisivas y radiales (Guerrero, 2006; Martín-Barbero, 1999; Winocur, 2003a, 2003b), se han complementado fuertemente por el aumento en la disponibilidad y la masificación en el uso de las tecnologías, que van moldeando un nuevo tipo de ciudadano el cual encuentra precisamente en los medios digitales, formas alternativas para ejercer sus derechos, informarse y participar en los asuntos públicos, llegando inclusive a constituirse como un nuevo tipo de ciudadanía denominado por algunos autores como ciudadanía digital, relacionada con el

conjunto de prácticas políticas y ciudadanas que de una forma u otra tratan de modificar y/o incidir en las instituciones, a través del uso de medios y tecnologías que tienen como característica la digitalización de sus mensajes y contenidos, como por ejemplo internet, pero 
también con otras tecnologías de información y comunicación (las llamadas TIC) como el teléfono celular" (Natal, Benítez, \& Ortiz, 2014, p. 9).

En términos generales, el ciudadano digital es aquel individuo que interactuando mediante una plataforma digital, puede ejercer su ciudadanía (Arcila, 2006), ya sea llevando a cabo unos cuantos aspectos, o bien la mayoría o todos de los elementos relacionados con los deberes y los derechos ciudadanos (Robles, 2009). En este sentido, es importante denotar que el ser o no ser ciudadano digital, está estrechamente vinculado con el "ser o no usuario de internet" (Robles, 2009, p. 8), por lo que para hablar del verdadero valor democrático de la ciudadanía digital, debería iniciar el análisis por la discusión de la cobertura de computadoras conectadas a internet, así como del análisis de las capacidades y destrezas digitales con las que cuente la población para poder sacarle utilidad a estas mismas herramientas digitales. En este punto conceptos como el de "brecha digital" (Carracedo, 2002; Castells, 2001, 2006; Hoffman, Novak, \& Schlosser, 2001; Servon, 2002), han intentado poner de relieve ese amplio porcentaje de la población, que por problemáticas de disponibilidad o de capacidad de uso, se encuentran aisladas de las ventajas que ofrecen las TIC.

Estas herramientas digitales también han sido cada vez más usadas por el gobierno y la administración pública para llevar a cabo actividades y mecanismos de gestión gubernamental, así como para ofrecer servicios públicos y gubernamentales. Debido a ello, es cada vez más común encontrarnos con conceptos como el de "gobierno digital" (Bertucci, 2005; Cortés, 2013; Sotelo, 2006); "gobierno electrónico" (Carter \& Belanger, 2004; Holden, Norris, \& Fletcher, 2003; Lenk \& Traunmüller, 2002); o quizá el que ha tenido mayor difusión, es decir, el "egobierno" (Aldrich, Bertot, \& McClure, 2002; Heeks \& Bailur, 2007; Kaufman, 2004; Moon, 2002). En términos generales, estos conceptos apuntan a la adopción de herramientas y plataformas digitales, por parte del gobierno, para abrir nuevas posibilidades en principalmente dos aspectos. En primer lugar para utilizarlos como herramientas para transparentar las acciones del gobierno y llevar a cabo la rendición cuentas. En segundo, con su utilización como mecanismos para llevar a cabo la ejecución propia de la administración pública, como lo pueden ser las áreas de comunicación social, prestación de servicios públicos, atención de usuarios o para brindar información general (Reilly \& Echeberría, 2003). Cabe hacer el señalamiento que como se vio, los conceptos relacionados con el e-gobierno se vinculan más como una forma diferente para llevar a cabo los procesos gubernamentales y de administración pública, sin que en ellos quede clara la importancia del uso de las tecnologías para promover una participación ciudadana más amplia.

Ahora bien, en términos generales la información permite a los ciudadanos alcanzar una serie de características específicas, en las que se encuentran:

1. Conocer de mejor forma su contexto político, social, cultural y económico específico, que les permita poder identificar problemáticas y retos que tiene su sociedad;

2. Poder argumentar de la mejor manera posible sus ideas, opiniones y posturas, mediante el dialogo, el discurso y la deliberación (Ganuza, 2012), y con ello tener una más eficiente aparición, en la llamada por algunos autores como la "esfera de lo público" (Arendt, 1993) o en el "mundo de la vida", conocido por algunos autores más (Habermas, 1987);

3. En la medida de lo posible, recortar las asimetrías sociales, dándole reconocimiento y voz a aquellos sectores de la sociedad que se encuentran en situación de vulnerabilidad, exclusión o atraso, pudiendo con ello alcanzar mejores niveles de desempeño social;

4. Derribar formas de gobierno altamente jerarquizadas, verticales o burocráticas, abriendo con ello formas de empoderamiento social, en donde ya no sólo la toma de decisiones públicas se realice desde los escritorios gubernamentales, sino desde los procesos de deliberación y llegada acuerdos mixtos entre los diferentes actores involucrados en un tema o en una política en específico;

5. Alcanzar niveles de gestión pública más amplios y democráticos, en donde los éxitos o fracasos de las políticas públicas son compartidos entre la sociedad y el gobierno, logrando con ello mejores niveles de trasparencia en la forma de toma de decisiones y de llevar a cabo los procesos públicos-gubernamentales. 
Algunos de estos elementos han sido reconocidos como principios fundamentales que dan pie tanto a la generación de estatutos jurisdiccionales, como por ejemplo el reconocimiento al acceso a la información enmarcado, desde el año de 1948, en el artículo 19 de la Declaración Universal de los Derechos Humanos, de la Organización de las Naciones Unidas (ONU); así como a principios tomados en consideración en estrategias de diversas agendas internacionales que persiguen niveles más amplios de desarrollo de las naciones.

En este punto, el Programa de las Naciones Unidas para el Desarrollo (PNUD) en el documento "El acceso a la información" del año 2013, menciona que para garantizar una sociedad democrática hace falta la real aplicación de los derechos vinculados a la libertad de expresión, así como al acceso a la información, pues estos derechos se constituyen como elementos fundamentales para garantizar tanto la capacidad de voz, como de participación de la ciudadanía. En este sentido, se hace mención a que igualmente importante resulta el fomentar y proteger el acceso a la información, así como también la construcción de mecanismos que permitan un flujo más oportuno de información entre los diversos actores involucrados en un tema específico, no quedándonos con exclusividad en entes gubernamentales, como lo son el propio gobierno o el parlamento, sino incluyendo a otros grupos como las organizaciones de la sociedad civil, el sector privado y a la ciudadanía en general. Estos flujos, según el PNUD, deberían reforzarse de forma específica en atención a aquellos sectores de la población que se encuentran en situaciones de vulnerabilidad y pobreza, con objeto de permitirles a estos grupos tener acceso a la posibilidad de influir en la construcción de las políticas y en general con el quehacer gubernamental (Programa de las Naciones Unidas para el Desarrollo, 2013).

Otro ejemplo que ilustra el interés por parte de los organizamos internacionales con relación al tema del acceso a la información, es el de los informes anuales del Banco Mundial sobre acceso a la información. En estos documentos este organismo ha intentado establecer ciertos principios fundamentales en el entendimiento de la información como un pilar muy importante para la construcción de ciudadanía. En el entendimiento de que la información permite tanto la rendición de cuentas, mejores prácticas gubernamentales centradas en la transparencia, así como una mayor eficiencia en el desempeño ciudadano. De igual forma este "acceso a la información crea nuevas oportunidades de uso de evidencias para encontrar y ofrecer soluciones locales innovadoras que permitan sacar a las personas de la pobreza" (Banco Mundial, 2013), coincidiendo este elemento con el tema de la importancia de la información como un principio muy importante con el cual, las personas en situación de pobreza, puedan verse ayudadas para alcanzar mejores niveles de desarrollo, así como mejores niveles de participación en la forma en la que son llevados los asuntos públicos.

En este contexto, se han buscado formas de gobierno que, basándose en niveles más amplios de información e intercambio de ideas, han pretendido alcanzar formas más plurales y horizontales tanto en la identificación de problemáticas, así como en la gestión de políticas públicas para darles solución. Una, que a últimos tiempos ha cobrado importante interés tanto por su tratamiento en los círculos de investigación especializada, así como por formar parte de muchas agendas de gobierno alrededor del mundo, es la del modelo denominado como gobierno abierto, mismo modelo que será abordado en el siguiente apartado de este trabajo, tomando en cuenta los pilares básicos que le constituyen, así como llevado a cabo su estudio a partir de su vinculación con el concepto de ciudadanía.

\section{La ciudadanía en el contexto del gobierno abierto}

Si bien es cierto que el concepto del gobierno abierto ha tenido un auge considerable en los últimos años, tanto en los debates de los círculos especializados de análisis, así como en los discursos políticos de muchas naciones (Coglianese, 2009; Ding et al., 2011; Lathrop \& Ruma, 2010; Lee \& Kwak, 2012; Yu \& Robinson, 2012), no es un término nuevo. Ejemplo de ello es que su uso inclusive ya había sido utilizado en el año de 1957 por Parks, para referirse a un tipo de derecho básico para los ciudadanos, vinculado a la necesidad de contar con información suficiente y pertinente sobre el quehacer gubernamental.

Sin embargo, el concepto adquiere amplia notoriedad a partir de una iniciativa gubernamental del presidente de los Estados Unidos de América, Barack Obama, quien realizando una crítica a la gestión pública que, hasta finales de la primera década del siglo XXI, había permeado la forma de hacer política; que tenía como referentes el establecimiento de los preceptos del New Public Management (Ferlie, 1996; Hood, 1995; McLaughlin, Osborne, \& Ferlie, 2002), el adelgazamiento sistémico del Estado y en general con la implementación de visiones gerenciales y de la iniciativa privada trasladados a la administración pública. A diferencia de lo anterior, con esta propuesta de Obama se pretendía alcanzar "un nivel de apertura en el gobierno sin precedentes y un sistema de transparencia, participación pública y colaboración que reforzara la democracia, asegurara la confianza pública y promoviera la eficacia y eficiencia gubernamental” (Obama, 2009). 
Para alcanzar lo anterior, el gobierno de los Estados Unidos de América impulsó la iniciativa "Alianza para el Gobierno Abierto" (AGA), que tuvo por objetivo el establecimiento y la adopción de ciertas prácticas de gestión gubernamental para hacer más eficientes a los gobiernos. Las primeras naciones en unirse a esta iniciativa, en el año 2011, fueron los gobiernos de México, Brasil, Filipinas, Indonesia, Noruega, Reino Unido, Sudáfrica y Tanzania; estas mismas naciones elaboraron un plan de acción particular, alineados a los cinco retos generales de la AGA (véase Tabla 1), los cuales tenían por objetivo alcanzar los "principios torales", mismos que pueden también definirse al tomar en consideración los pilares conceptuales que le dan sustancia a la idea del gobierno abierto, que son: a) la transparencia y la rendición de cuentas, b) la participación ciudadana y c) la innovación y la tecnología.

\begin{tabular}{|l|l|}
\hline \multicolumn{1}{|c|}{ Reto } & \multicolumn{1}{|c|}{ Contenido } \\
\hline Mejora de servicios públicos & $\begin{array}{l}\text { Comprende toda la gama de servicios públicos a la } \\
\text { población, tales como salud, educación, justicia penal, agua } \\
\text { potable, electricidad y telecomunicaciones y se busca } \\
\text { promover la mejora de los servicios públicos o bien la } \\
\text { innovación por parte del sector privado. }\end{array}$ \\
\hline Aumentar la integridad pública & $\begin{array}{l}\text { Ética pública, prevención y ataque a la corrupción, acceso a } \\
\text { la información, reformas financieras, así como promoción y } \\
\text { afianzamiento de libertades de la sociedad civil y de los } \\
\text { medios de comunicación. }\end{array}$ \\
\hline $\begin{array}{l}\text { Manejo de recursos públicos con mayor eficacia y y } \\
\text { eficiencia }\end{array}$ & $\begin{array}{l}\text { Asignación de recursos presupuestarios, financiamiento } \\
\text { tanto interno como externo y aprovechamiento y } \\
\text { conservación de recursos naturales. }\end{array}$ \\
\hline Construcción de comunidades más seguras & $\begin{array}{l}\text { Seguridad pública, respuesta y atención ante desastres } \\
\text { naturales, riesgos ambientales y protección civil }\end{array}$ \\
\hline $\begin{array}{l}\text { Aumento de la rendición de cuentas por parte de } \\
\text { las empresas }\end{array}$ & $\begin{array}{l}\text { Responsabilidad empresarial en temas tales como medio } \\
\text { ambiente, protección del consumidor, participación de la } \\
\text { comunidad y lucha anticorrupción. }\end{array}$ \\
\hline
\end{tabular}

Tabla 1 - Grandes Retos de la Alianza para el Gobierno Abierto según la propia AGA

En este mismo punto, algunos autores como Blomgren y Foxworthy (2014) han hecho hincapié en estos principios constitucionales del concepto del gobierno abierto, vinculando su importancia al funcionamiento ciudadano. En este sentido, se ha mencionado que la transparencia puede llegar a desatar procesos de rendición de cuentas, que a su vez empoderen mediante la información a la ciudadanía; y con ello se puedan alcanzar mejores niveles tanto en el funcionamiento gubernamental así como en la participación ciudadana, pues mediante el intercambio de ideas, posturas y visiones que permitan una colaboración entre los diversos actores involucrados en un tema en específico, se lograría alcanzar un mejor funcionamiento del desempeño gubernamental y del desempeño social.

En este contexto, se considera pertinente abordar de forma específica cada uno de los pilares conceptuales, con objeto de conocer su vinculación específica con el concepto de ciudadanía. En primer lugar se encuentran los conceptos de la transparencia y la rendición de cuentas, este último elemento vinculado de forma directa con el accountability (Akutsu \& Pinho, 2003; Peters, 1998), pues toman vigencia en los debates sobre el aumento de los valores democráticos de los países, en donde se toma en consideración la necesidad de que tanto los gobiernos, así como los propios funcionarios públicos, informen amplia y oportunamente a la ciudadanía, sobre las decisiones y las justificaciones en la forma en que son tratados los asuntos públicos. Con esta información se espera que la ciudadanía tenga por lo menos dos elementos positivos para ampliar su participación, en primer lugar contar con elementos informativos para aprobar o sancionar el actuar gubernamental y de los funcionarios públicos, y el segundo para que la ciudadanía tenga oportunidad de participar directamente, mediante la deliberación y la concertación de ideas, en la forma específica en la que son atendidos los asuntos públicos. 
En este mismo sentido, autores como Cunill Grau (2006), bajo el análisis del principio de la transparencia como valor fundamental de la democracia para los países latinoamericanos, han mencionado que "buena parte de las leyes de la región que expresamente garantizan el libre acceso a la información pública han sido promovidas por organizaciones de la sociedad civil, en particular, de medios de comunicación que reivindican la circulación de la información como vehículo de libertad" (Cunill Grau, 2006, p. 23). Sin embargo, es oportuno mencionar que la información por sí misma no se traduce necesariamente en transparencia, sino que debe poseer una serie de características específicas para que alcance ese estatus, como por ejemplo el que sea oportuna, relevante, verídica y amplia, con objeto de que los diversos actores involucrados, entre ellos la ciudadanía, en un tema público en común, puedan desarrollar sus valoraciones y posicionamientos con respecto a la forma en la que se está atendiendo el propio asunto público. Kaufmann (2005), ahondando en este tema, ha señalado que para que la información pueda dar como resultado la transparencia necesita de por lo menos cuatro características básicas, que son: la accesibilidad, la relevancia, la confiabilidad y la calidad.

Otro concepto esencial en la idea del gobierno abierto es el de la participación ciudadana, que se debería de dar en formas más plurales y horizontales en la manera en la que son atendidos los asuntos públicos y elaboradas las políticas, es decir, en formas de gobierno más ligadas a la gobernanza (Aguilar-Villanueva, 2006; Mayntz, 2000, 2002; Peters, 1998; Scharpf, 2000, 2001). En este sentido, la perspectiva del gobierno abierto intenta alejarse de las visiones y contradicciones en las que cayó el modelo de gobernabilidad (Camou, 1995, 2000; Uvalle, 2008), que en los últimos tiempos se le asocia más con su contraparte negativa, es decir la ingobernabilidad (Coppedege, 1996), relacionada con problemáticas de sobrecarga económica y de servicios gubernamentales, así como por la existencia de un gran cúmulo de demandas públicas insatisfechas, por la incapacidad gubernamental de atenderlas adecuadamente (Pasquino, 2005).

En este punto es preciso hacer una breve diferenciación conceptual entre los términos gobernabilidad y gobernanza con objeto de clarificar el uso del concepto de la gobernanza en el análisis del gobierno abierto. En este sentido, la gobernabilidad se relaciona en general con la capacidad gubernamental para alcanzar niveles de legitimidad, y por ende, en intentar alcanzar mayores niveles democráticos que permitan a los gobiernos implementar políticas y mantener un orden, aceptación y estabilidad social y política. Por otro lado, el concepto de gobernanza se vincula de forma más directa con el binomio reglas del juego/resultados gubernamentales, en donde se destacan más aspectos como la capacidad de generar escenarios más colaborativos y participativos en la gestión de las políticas públicas; es decir, se centra más en el aspecto de la coordinación multinivel de diversos actores, que mediante su participación directa en la elaboración de políticas, lleven a acuerdos plurales en la forma en la que son atendidos los asuntos públicos.

En este contexto, el modelo de la gobernanza adoptado en la idea del gobierno abierto, se vincula más con estos aspectos no sólo relacionados con el restablecimiento de un equilibrio político mediante la apertura de oportunidades de participación ciudadana, sino también con el objetivo de alcanzar mejores niveles de gestión pública (más eficientes y eficaces), mediante la apertura de información pública relevante, la mejora del funcionamiento de los servicios públicos y el establecimiento de mecanismos de coordinación de actores que limiten, en mayor o menor medida, los desacuerdos, rupturas y enfrentamientos entre los diversos actores, tanto gubernamentales como sociales, interesados en un tema público común (Heritier, 2001; Mayntz, 2000; Scharpf, 2000).

Finalmente, un tercer pilar en el que se sostiene la idea del gobierno abierto es el de la utilización de las TIC, las herramientas digitales e internet, para llevar a cabo tanto los procesos que permitan alcanzar mayores niveles de transparencia y rendición de cuentas en la forma en la que son atendidos los asuntos públicos, así como medios fundamentales para aumentar las posibilidades de participación de la ciudadanía en la gestión de las políticas públicas. Esta utilización de las herramientas digitales en la forma de llevar a cabo los procesos de gobierno, apuntarían a la idea de una innovación sistémica o transformacional, descrita por Ramírez-Alujas, en donde este tipo de innovaciones "dan lugar a nuevas estructuras en la fuerza de trabajo y nuevas formas y tipos de organización, transformando sectores enteros, y cambiando dramáticamente las relaciones interorganizacionales y el desempeño general” (2010, p. 10).

En este punto, el cambio de paradigma que va de un gobierno tradicional al gobierno abierto, apunta no sólo a la adopción de tecnologías, sino a una transformación mayor en la manera de llevar a cabo los procesos gubernamentales, y a la apertura de canales más amplios de participación ciudadana para la toma de decisiones públicas. 
Tomando en consideración todos estos elementos, es de destacar cómo el elemento de la biblioteca pública, lugar por excelencia para la obtención y preservación de la información, ha estado ausente en la implementación de los mecanismos del gobierno abierto de algunos países, como lo es en el caso específico de México. En este sentido, en el último apartado de este documento se pone a consideración el tema del papel de la biblioteca pública en el contexto del gobierno abierto, partiendo de su perspectiva como espacio democrático en el cual se pueden reforzar pertinentemente los postulados del gobierno abierto.

\section{Potencialidades de la biblioteca pública en el gobierno abierto}

Si bien la biblioteca es por excelencia el lugar en el que los individuos pueden acceder a información útil y relevante que les permita alcanzar, no sólo un mayor nivel de conocimientos, sino también de vida, la temática ha estado olvidada en el desarrollo de los esfuerzos gubernamentales de muchos países involucrados en la AGA, como es el caso de México. Por lo tanto, se torna fundamental rescatar algunos de los elementos propuestos por autores que han abordado la temática de la importancia de la biblioteca pública como un elemento esencial para alcanzar un mejor desempeño social.

En este sentido, se podría partir de la premisa que apunta a la necesidad de "que la biblioteca se proyecte hacia afuera y asuma el papel que le corresponde en la educación, en la enseñanza y en la formación y difusión de los recursos culturales del país" (Suaiden, 2002, p. 333). Esta premisa se inscribe en el entendimiento de la información como un elemento que genera poder a los individuos: poder relacionado con la capacidad de llegar a tomar las mejores decisiones posibles para alcanzar mejores y mayores niveles de desarrollo, poder relacionado con la capacidad de influir en la forma en la que se atienden los asuntos públicos y la política en general, así como poder, que transformado en conocimiento, puede llegar a conformar la llamada sociedad del conocimiento para la generación de producción, servicios y en general la riqueza de las naciones.

De igual forma, existe un estrecho vínculo entre las funciones de la biblioteca pública y la profundización y consolidación de los valores democráticos de los países, pues inclusive Grattan (1938), hace ya casi un siglo que visualizó a las bibliotecas como instituciones fundamentales para la democracia. En este mismo sentido se ha mencionado que la biblioteca puede y debe de llegar en sí misma a ser un espacio democrático, pero para llegar a serlo hace falta que se cumplan tres requisitos previos, los que a su vez, deben estar a cargo de las actividades o quehaceres del

bibliotecario público: en primer lugar promover la lectura como una práctica social y cultural; en segundo lugar garantizar el acceso a la información internacional, nacional y local en múltiples soportes y formatos, esto desde una visión democratizante y equitativa; y, en tercer lugar, divulgar las manifestaciones artísticas y culturales, para contribuir a la construcción de una cultura propia con sentido universal (Betancur, 2002, p. 3).

Desde estas perspectivas se deja de manifiesto que no puede existir una verdadera democracia sin una real participación ciudadana de forma activa, y no se puede ejercer una verdadera participación activa, si no se tiene información relevante y útil que haga posible esa participación.

De lo anterior se desprende la necesidad de que en las discusiones sobre la temática del gobierno abierto, se rescate la importancia de la biblioteca pública como una institución muy útil en su dimensión facilitadora de información relevante y trascendental, con la que los individuos pueden alcanzar un crecimiento intelectual (Sharma, 1987), así como formarse opinión, y de esta forma, ejercer de mejor forma el derecho a la participación en el contexto democrático de las naciones. Por ello, en un primer momento, se torna fundamental que las instancias gubernamentales pongan en marcha la implementación de políticas y programas que tengan por objetivo el fortalecimiento y mejora de estos espacios públicos.

A la par de este entendimiento de las bibliotecas públicas como espacios para la búsqueda y obtención de información útil o socialmente importante y trascendente, también se pueden llegar a consolidar, siguiendo los aportes de Ford (2002), como "terceros lugares", es decir, espacios que vayan más allá de lo privado que se da en el hogar o de lo que ocurre en el trabajo, y que tengan como característica la apertura para que las personas puedan entrar en contacto, conocerse y así generar un sentimiento de comunidad, con lo cual se verían reforzados los lazos de solidaridad de los ciudadanos de un lugar específico, pues como la propia autora lo sostiene

la actividad que sustenta estos "terceros espacios" suele ser la conversación. Los terceros

lugares son espacios que acogen a poblaciones diversas, donde se desarrollan 
conversaciones impredecibles y donde surge la novedad, fruto de la capacidad creativa agregada del colectivo. Las afinidades que se derivan de estas asociaciones casuales e informales contribuyen a crear comunidades fuertes (Ford, 2002, p. 191).

Tomando en consideración esta perspectiva, sería pertinente impulsar el surgimiento de bibliotecas públicas como "terceros lugares", en las que se promuevan el encuentro, la comunicación y el contacto, no exclusivamente entre personas con semejantes formas de pensamiento, sino, tomando en consideración su característica de apertura a toda la sociedad, de formas de pensamiento plurales y diversas, que enriquezcan y generen mejores niveles de los lazos de pertenencia de los individuos para con la colectividad; lo que podría traer a su vez mejores niveles de cohesión y concertación social.

Otra perspectiva igualmente importante es la de tomar en consideración a las bibliotecas públicas desde una dimensión política a partir de una perspectiva de pedagogía social. En este sentido se ha mencionado que resulta muy importante para la sociedad que la biblioteca pública sea considerada "como espacio de construcción de la identidad y el reconocimiento (elementos claves para la ciudadanía), para la participación consciente y en igualdad de condiciones y de oportunidades, y no sólo como complemento de la escolaridad" (Jaramillo, 2010, p. 289). Esta perspectiva sobrepasa la visión y el entendimiento de las bibliotecas como meras acompañantes del proceso de educación escolar, dimensión que por demás es importante tomar en consideración en los análisis, sino que se les atribuye aspectos de identidad, encuentro y compromiso frente a la comunidad, no solo de una clase social en particular, es decir no desde un entendimiento limitativo, sino de apertura y plural, en la que los individuos que se han visto excluidos históricamente de los canales de participación, puedan adquirir conocimientos y voz que ayude a su vez a obtener una más equilibrada justicia social.

De igual forma, en el entendimiento del gobierno abierto, como se vio anteriormente, se destaca el tema de la democratización del acceso a la información, puesto que la información puede dotar a los individuos, entre otras cosas, de cultura, educación y conocimientos, mismos elementos que terminan teniendo relación con la capacidad reflexiva y crítica de los individuos sobre el desarrollo de los acontecimientos sociales, así como su capacidad de argumentación de propuestas e ideas que les permitan participar de forma directa en esos mismos acontecimientos. Desde esta perspectiva, se debe entender a la información como un derecho fundamental para el buen desarrollo ciudadano, pues se constituye como "un derecho fundamental para el ejercicio de los derechos civiles, jurídicos y sociales que contribuye a reducir la brecha entre informados y desinformados" (Jaramillo, 2010, p. 289). Estos elementos inscriben a la biblioteca pública como una institución caracterizada por un ambiente de pluralidad y respeto, que a fin de cuentas favorece el encuentro de los ciudadanos y por ende puede fungir como una instancia mediadora para la construcción de ciudadanía (Giraldo \& Román, 2011).

Otro elemento interesante en este análisis es el de la educación social que puede ser promovida desde la biblioteca pública (Jaramillo, 2011, 2013; Jaramillo \& Quiroz-Posada, 2013), pues resulta en otro factor que favorece la construcción ciudadana, sobre todo en aquellos países en donde los retos de la calidad y cobertura de la educación son temas constantes de las agendas públicas por los problemas estructurales en materia de educación. En este sentido, se puede visualizar a la biblioteca pública como un lugar que les permite a los individuos obtener conocimientos, educación, habilidades y destrezas más allá de las que pueden obtener en las aulas escolares. Bajo esta perspectiva, las personas que por diferentes circunstancias o razones no hayan tenido acceso a la educación formal, pueden contar con un espacio en el que les es posible obtener información y conocimientos, que a final de cuentas, redundan en otros canales de formación y educación. Sin embargo, para que estas posibilidades se vean materializadas en la realidad, se torna fundamental que exista un compromiso gubernamental amplio, que se vea reflejado en la gestión de políticas públicas para el desarrollo de las colecciones de las bibliotecas públicas, así como con la correcta capacitación de las personas que brindan los servicios bibliotecarios, temas que son fundamentales para que las posibilidades de la educación social, desde la biblioteca pública, tengan realmente viabilidad.

Un último elemento que es pertinente tomar en cuenta en este apartado, es el relacionado con el tema, cada vez más tomado en consideración, relacionado con la necesidad de entender a la ciudadanía desde una perspectiva amplia, en donde todas las voces posibles pueden redundar en la construcción de un pilar esencial en el que se sostenga la democracia. Esta amplia variedad tanto de información como de puntos de vista, han sido englobados en algunos trabajos bajo el concepto de "infodiversidad", la cual 
es pluralidad, es rescate, es conservación, es disponibilidad y libre acceso a la información. La infodiversidad es el conjunto de acciones y funciones que aseguran a todo ser humano la posibilidad de vivir en un ambiente de fuerzas y productos sociales que lo enriquecen con la diversidad de ideas y pensamientos del pasado y del presente, del norte y del sur, del oriente y del occidente y que establecen un equilibrio en su vida como individuo y en la del grupo social al que pertenece (Morales, 2001, pp. 43-44).

De igual forma, en el entendimiento del gobierno abierto, resulta fundamental tomar en cuenta esa pluralidad y diversidad de puntos de vista, que pueden llegar a enriquecer considerablemente la gestión de las políticas, así como la implementación de programas e iniciativas para atender los asuntos públicos, pues como ya se ha mencionado, una de las premisas fundamentales del gobierno abierto es precisamente la de la necesidad de la apertura en los procesos para alcanzar acuerdos en las formas en las que se llevaran a cabo los procesos gubernamentales. En este punto, el apoyo tanto monetario como logístico del gobierno o de las agencias tanto públicas como privadas, para con el impulso de las actividades de la biblioteca pública resulta fundamental. Solo de esta forma las bibliotecas públicas podrían tener un adecuado desarrollo de colecciones que resulten pertinentes para su comunidad, así como contar con documentos en múltiples formatos (escritos, sonoros, fotográficos y/o audiovisuales), que tengan por objetivo brindar elementos fundamentales en los que se base la participación de una ciudadanía activa, así como involucrada constantemente con su devenir histórico y político.

\section{Consideraciones finales}

En el presente documento se abordo el análisis de la ciudadanía a partir de la importancia que, para la construcción de la misma, tiene la información. En este contexto se puede argumentar que para la construcción de cualquier tipo de ciudadanía (ya sea pasiva o participativa) es necesaria la información. Para la ciudadanía pasiva o de baja intensidad, relacionada principalmente con la capacidad de elegir a los representantes del gobierno mediante la emisión del voto, resulta esencial contar con información relativa al perfil o al desempeño en la gestión pública de los candidatos a ocupar cargos de representación popular, así como con la información relativa a las propuestas, iniciativas y programas que los mismos candidatos propongan desarrollar en sus gestiones. Con mucho mayor peso, el ciudadano, en su entendimiento participativo o deliberativo, necesita información útil, vasta, relevante y completa, que le permita conocer el contexto general de los asuntos públicos así como argumentar de forma correcta sus puntos de vista y sus sugerencias en la forma en la que se lleva a cabo la política.

Iniciativas gubernamentales alrededor del mundo han destacado la necesidad de contar con una mayor vinculación de los ciudadanos en la forma en la que son tratados los temas de interés general y con la política. Una de estas iniciativas es la del gobierno abierto, como lo deja de manifiesto la creación de la "Alianza para el Gobierno Abierto", que impulsada desde el año 2011 por el gobierno de los Estados Unidos de América, más otros siete gobiernos en los que se incluyen los de México y Brasil, cuenta en la actualidad con la suma de 65 países que tomando en consideración la Declaración del Gobierno Abierto internacional, construyen sus propios planes de acción para alcanzar los ideales perseguidos por el gobierno abierto.

Estos ideales están relacionados con los pilares conceptuales que le dan cuerpo al propio concepto de gobierno abierto, los cuales son: 1) contar con una mayor transparencia así como con una mejor rendición de cuentas, impulsando mecanismos para que la información pública-gubernamental sea de fácil acceso a todos los ciudadanos; 2) alcanzar mejores niveles de participación ciudadana, acercándose al modelo de la gobernanza, traducida como la coordinación de distintos actores multinivel interesados en un problema o una política en concreto; y 3) utilizar a las tecnologías de la información y comunicación tanto para acercar al gobierno con los ciudadanos, así como para establecer nuevos mecanismos de información, empoderamiento y capacidad de voz a los ciudadanos.

En este sentido, un tema pendiente por revalorar en las discusiones del gobierno abierto en muchas naciones, como lo es el caso específico de México, es el de impulsar el desarrollo de las bibliotecas públicas como espacios en los que por excelencia se puede acceder a información socialmente relevante.

Existen ejemplos muy interesantes que ilustran el papel de las bibliotecas públicas como espacios de formación ciudadana y de activación de la participación ciudadana. Los registros encontrados en las bibliotecas públicas de Antioquia, Colombia, muestran cómo estas instituciones pueden llegar a consolidarse como "instituciones sociales que cumplen una tarea fundamental de estímulo al desarrollo local en perspectiva global, de formación 
de ciudadanos con valoraciones y actitudes positivas frente a la información, desde su consumo crítico" (Betancur, 2002, p. 42).

Finalmente, es importante que se activen programas o políticas que permitan la integración de la biblioteca pública como un elemento esencial en los temas del gobierno abierto, lo cual no debe ser sólo materia de reflexión académica, sino que también de acción de gobierno. En este contexto, es necesario que los bibliotecólogos así como los especialistas de la información tomen cada vez un papel más protagónico en la discusión de los temas del gobierno abierto, como actores fundamentales, tanto por sus conocimientos así como por sus habilidades, en la discusión de los rumbos que sigan las políticas y programas públicos para generar mayores niveles de participación ciudadana, recordando que el fin último que persiguen los ideales como los del gobierno abierto, son los de alcanzar una más amplia cohesión social, mejorar el actuar gubernamental, así como obtener mayores niveles democráticos y de desarrollo de las naciones.

\section{Referencias}

Aguilar-Villanueva, L. F. (2006). Gobernanza y gestión pública. México D.F.: Fondo de Cultura Económica.

Akutsu, L., \& Pinho, J. A. (2003). Sociedade da informação, accountability e democracia delegativa: investigação em portais de governo no Brasil. Revista de Administração Pública, 36(5), 723-746.

Aldrich, D., Bertot, J. C., \& McClure, C. R. (2002). E-Government: Initiatives, Developments, and Issues. Government Information Quarterly, 19(4), 349-55.

Arcila, C. (2006). El ciudadano digital. Revista Latinoamericana de Comunicación CHASQUI, (93), 18-21.

Arendt, H. (1993). La condición humana. Barcelona: Paidós.

Banco Mundial. (2013). Informe anual sobre acceso a la información del Banco Mundial.

Bertucci, G. (2005). Gobierno digital y valor público. Política digital, (23), 12-14.

Betancur, A. (2002a). La biblioteca pública en la perspectiva del desarrollo local: una estrategia para la democracia (p. 8). Presentado en 68th IFLA Council and General Conference, Glasgow: IFLA.

Betancur, A. (2002b). Un lugar en el mundo: Los servicios de información local en la biblioteca pública. Métodos de Información, 9(51), 38-43.

Blomgren, L., \& Foxworthy, S. (2014). Collaborative Governance and Collaborating Online: The Open Government Initiative in the United States. En E. Bohne, J. D. Graham, J. Raadschelders, \& P. Lehrke (Eds.), Public Administration and the Modern State. Nueva York: Palgrave Macmillan.

Bolos, S. (Ed.). (2008). Mujeres y Espacio Público: construcción y ejercicio de la ciudadanía. México: Universidad Iberoamericana.

Bonet, A. J. A. (2009). La ciudadanía como proceso de emancipación: Retos para el ejercicio de ciudadanías de alta intensidad. Astrolabio: revista internacional de filosofia, (9), 13-24.

Borchorst, N. G., Bødker, S., \& Zander, P.-O. (2009). The boundaries of participatory citizenship. En I. Wagner, H. Tellioğlu, E. Balka, C. Simone, \& L. Ciolfi (Eds.), ECSCW 2009 (pp. 1-20). Londres: Springer London.

Camou, A. (1995). Gobernabilidad y democracia. México D.F.: Instituto Federal Electoral.

Camou, A. (2000). La múltiple (in) gobernabilidad: elementos para un análisis conceptual. Revista Mexicana de Sociología, 62(4), 159-188.

Carracedo, J. D. (2002). Jerarquías y desigualdades en la sociedad de la información: la estratificación digital en relación con la democracia digital. En H. Cairo (Ed.), Democracia digital: límites y oportunidades. Madrid: Trotta.

Carter, L., \& Belanger, F. (2004). Citizen adoption of electronic government initiatives. En Proceedings of the 37th Annual Hawaii International Conference on System Sciences, 2004 (p. 10 pp.-pp.). Hawaii.

Castells, M. (2001). La Galaxia Internet. Barcelona: Plaza y Janés.

Castells, M. (2006). La sociedad red: una visión global. Madrid: Alianza Editorial. 
Coglianese, C. (2009). The Transparency President? The Obama Administration and Open Government. Governance, 22(4), 529-544.

Coppedege, M. (1996). El concepto de gobernabilidad. Modelos positivos y negativos. En Ecuador: un problema de gobernabilidad. Quito: CORDES-PNUD.

Correa, E., \& Noé, M. (Eds.). (1998). Nociones de una ciudadanía que crece. Santiago de Chile: FLACSO-Chile.

Cortés, R. (2013). Análisis comparado de las políticas públicas de gobierno digital en Costa Rica y Chile entre los años 2002 y 2010. San José: Universidad de Costa Rica.

Cunill Grau, N. (2006). La Transparencia en la Gestión Pública: ¿Cómo construirle viabilidad? Estado, gobierno, gestión pública: Revista Chilena de Administración Pública, (8), 22-44.

Dasí, J. (2005). Nuevas formas de gobernanza para el desarrollo sostenible del espacio relacional. Ería, 67, 219-235.

Ding, L., Lebo, T., Erickson, J. S., DiFranzo, D., Williams, G. T., Li, X., ... Hendler, J. A. (2011). TWC LOGD: A portal for linked open government data ecosystems. Web Semantics: Science, Services and Agents on the World Wide Web, 9(3), 325-333.

Farinós, J. (2005). Nuevas formas de gobernanza para el desarrollo sostenible del espacio relacional. Ería, (67), 219-235.

Ferlie, E. (1996). The New Public Management in Action. Oxford University Press.

Fleury, S. (2003). La expansión de la ciudadanía. VVAA: Inclusión social y nuevas ciudadanías. Bogotá: Pontifica Universidad Javeriana.

Ford, B. J. (2002). Todos son bienvenidos: la biblioteca pública como espacio de integración ciudadana. En I Congreso Nacional de Bibliotecas Públicas (pp. 191-200).

Ganuza, E. (2012). El desafío deliberativo. En I. Ramos \& E. Campos (Eds.), Ciudadanía en 3D: democracia digital deliberativa. Un análisis exploratorio (pp. 19-52). Barcelona: Edhasa.

Giraldo, Y., \& Román, G. E. (2011). La biblioteca pública como mediadora en la construcción de la ciudadanía. Em Questão, 17(1), 211-230.

Grattan, H. (1938). Libraries: a necessity for democracy. Sydney: Free Library Movement.

Guerrero, M. (2006). Medios de comunicación y la función de transparencia. México D.F.: Instituto Federal de Acceso a la Información Pública.

Habermas, J. (1987). Teoría de la acción comunicativa. Volumen 2: Crítica de la razón funcionalista. Madrid: Taurus.

Habermas, J. (1998). Facticidad y validez. Madrid: Trotta.

Heeks, R., \& Bailur, S. (2007). Analyzing e-government research: Perspectives, philosophies, theories, methods, and practice. Government Information Quarterly, 24(2), 243-265.

Heritier, A. (2001). New Modes of Governance in Europe: Policy-Making without Legislating? (SSRN Scholarly Paper No. ID 299431). Rochester, NY: Social Science Research Network.

Hoffman, D. L., Novak, T. P., \& Schlosser, A. E. (2001). The evolution of the digital divide: examining the relationship of race to internet access and usage over time. En B. M. Compaine (Ed.), The Digital Divide (pp. 47-97). Cambridge: MIT Press.

Holden, S., Norris, D. F., \& Fletcher, P. D. (2003). Electronic Government at the Local Level. Public Performance \& Management Review, 26(4), 325-344.

Hood, C. (1995). The "new public management" in the 1980s: Variations on a theme. Accounting, Organizations and Society, 20(2-3), 93-109.

Jaramillo, O. (2010). La biblioteca pública, un lugar para la formación ciudadana: referentes metodológicos del proceso de investigación. Revista Interamericana de Bibliotecología, 33(2), 287-313.

Jaramillo, O. (2011). La biblioteca pública, lugar para la construcción de ciudadanía: una mirada desde la educación social (Tesis de doctorado). Facultad de Educación, Universidad de Antioquia, Medellín.

Jaramillo, O. (2013). Biblioteca pública, ciudadanía y educación social. Buenos Aires: Alfagrama Ediciones.

Jaramillo, O., \& Quiroz-Posada, R.-E. (2013). La educación social dinamizadora de prácticas ciudadanas en la biblioteca pública. Revista Educação \& Sociedade, 34, 139-154. 
Kaufman, E. (2004). E-gobierno en Argentina: crisis, burocracia y redes. En R. Araya \& M. A. Porrúa (Eds.), América Latina puntogob. Casos y tendencias en gobiernos electrónicos. Santiago de Chile: Flacso Chile.

Kaufmann, D. (2005). Transparency Matters: the "Second Generation" of Institutional Reform. Washington: Development Gateway Foundation.

Kymlicka, W., \& Norman, W. (1997). El retorno del ciudadano. Una revisión de la producción reciente en teoría de la ciudadanía. Agora, 7, 5-42.

Lathrop, D., \& Ruma, L. (2010). Open Government: Collaboration, Transparency, and Participation in Practice. O'Reilly Media, Inc.

Lechner, N. (2000). Nuevas Ciudadanías. Revista de estudios sociales, (5), 25-31.

Lee, G., \& Kwak, Y. H. (2012). An Open Government Maturity Model for social media-based public engagement. Government Information Quarterly, 29(4), 492-503.

Lenk, K., \& Traunmüller, R. (2002). Electronic Government: Where Are We Heading? En R. Traunmüller \& K. Lenk (Eds.), Electronic Government (pp. 1-9). Springer Berlin Heidelberg.

Martín-Barbero, J. (1999). El miedo a los medios. Política, comunicación y nuevos modos de representación. Nueva Sociedad, (161), 43-56.

Mayntz, R. (2000). Nuevos desafíos de la teoría de Governance. Instituciones y desarrollo, (7), 1-10.

Mayntz, R. (2002). National states and global governance. En II Inter-American Congress of CLAD on State and Public Administration Reform Lisbon, Portugal (pp. 1-8). Lisboa: CLAD.

McLaughlin, K., Osborne, S. P., \& Ferlie, E. (2002). New Public Management: Current Trends and Future Prospects. Psychology Press.

Moon, M. J. (2002). The Evolution of E-Government among Municipalities: Rhetoric or Reality? Public Administration Review, 62(4), 424-433

Morales, E. (2001). La diversidad informativa latinoamericana en México. Centro Coordinador y Difusor de Estudios Latinoamericanos - UNAM.

Natal, A., Benítez, M., \& Ortiz, G. (2014). Ciudadanía digital. México D.F.: Juan Pablos.

Obama, B. (2009). Memorándum sobre Transparencia y Gobierno Abierto.

O’Donnell, G. (1993). Estado, Democratización y ciudadanía. Nueva Sociedad, (128), 62-87.

O’Donnell, G. (1994). Delegative Democracy. Journal of Democracy, 5(1), 55-69.

Parker, W. C. (1989). Participatory Citizenship: Civics in the Strong Sense. Social Education, 53(6), 353-54.

Parks, W. (1957). Open Government Principle: Applying the Right to Know Under the Constitution. George Washington Law Review, 26, 1.

Pasquino, G. (2005). Gobernabilidad. N. Bobbio, N. Matteucci, \& G. Pasquino (Eds.), Diccionario de Política (pp. 703-710). Madrid: Siglo XXI.

Peruzzotti, E. (1997). La naturaleza de la nueva democracia argentina: revisando el modelo de democracia delegativa (Tema Central). Ecuador Debate, (42), 130-147.

Peters, B. G. (1998). R. A. W. Rhodes, Understanding Govenance: Policy Networks, Governance, Reflexivity and Accountability. Public Administration, 76(2), 408-409.

Programa de las Naciones Unidas para el Desarrollo. (2013). El acceso a la información.

Ramírez-Alujas, Á. (2010). Innovación en la Gestión Pública y Open Government (Gobierno Abierto): Una Vieja Nueva Idea. Revista Buen Gobierno, (9), 35.

Reilly, K., \& Echeberría, R. (2003). El papel del ciudadano y de las OSC en el e- Gobierno. Un estudio de gobierno electrónico en ocho países de América Latina y el Caribe. Quito: Monitor de políticas TIC y derechos en internet en América Latina y el Caribe.

Robles, J. M. R. (2009). Ciudadanía digital: Una introducción a un nuevo concepto de ciudadano. Barcelona: Editorial UOC. 
Scharpf, F. W. (2000). Economic Changes, Vulnerabilities, and Institutional Capabilities. En F. W. Scharpf \& V. A. Schmidt (Eds.), Welfare and Work in the Open Economy Volume I: From Vulnerability to Competitiveness in Comparative Perspective (pp. 21-124). Oxford University Press.

Scharpf, F. W. (2001). European Governance: Common Concerns vs. The Challenge of Diversity. Jean Monnet Working Paper.

Servon, L. (2002). Bridging the Digital Divide. Technology, community and public policy. Oxford: Blackwell Publishing.

Sharma, P. (1987). Libraries and Society (Ess Ess Publications). Nueva Delhi.

Sotelo, A. (2006). México: gobierno digital en expansión. México D.F.: Edición del autor.

Suaiden, E. J. (2002). El impacto social de las bibliotecas públicas. Anales de Documentación, 5(0), 333-344.

Uvalle, R. (2008). Gobernabilidad, transparencia y reconstrucción del Estado. Revista Mexicana de Ciencias Políticas y Sociales, $L(208)$, 97-116.

Valente, V. (2000). Una reflexión feminista de la ciudadanía. Estudios feministas, 8(2), 17.

Vromen, A. (2003). People Try to Put Us Down ...: Participatory Citizenship of "Generation X". Australian Journal of Political Science, 38(1), 79-99.

Winocur, R. (2003a). Ciudadanos en los medios. La construcción de lo público en la radio. Buenos Aires: Gedisa.

Winocur, R. (2003b). La invención mediática de la ciudadanía. En P. Ramírez (Ed.), Espacio público y reconstrucción de ciudadanía (pp. 231-252). México D.F.: Miguel Ángel Porrúa.

Yu, H., \& Robinson, D. G. (2012). The New Ambiguity of "Open Government" (SSRN Scholarly Paper No. ID 2012489). Rochester, NY: Social Science Research Network.

\section{Datos del autor}

\section{Alejandro Ramos Chávez}

Investigador del Instituto de Investigaciones Bibliotecológicas y de la Información, Universidad Nacional Autónoma de México

aramos@iibi.unam.mx

Recibido - Received: 2016-08-23

Aceptado - Accepted: 2016-11-17

\section{(cc) $\mathrm{EY}$}

This work is licensed under a Creative Commons Attribution 4.0

United States License.

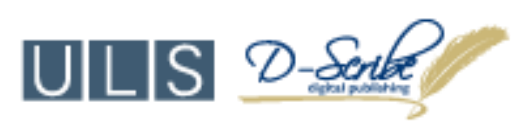

This journal is published by the University Library System of the University of Pittsburgh as part of its D-Scribe Digital Publishing Program and is cosponsored by the University of Pittsburgh Press. 